\title{
Análisis Psicométrico y Adaptación del Test de Autoconcepto Forma 5 en Universitarios Asiduos al Uso de Videojuegos
}

\author{
Psychometric Analysis and Adaptation of the Self-Concept Test (Form 5) on \\ University Students Who Play Video Games Frequently
}

\author{
Asunción Martínez Martínez ${ }^{1}$, Félix Zurita Ortega ${ }^{2}$, Ramón Chacón Cuberos ${ }^{3}$, Tamara Espejo \\ Garcés $^{4}$, Manuel Castro Sánchez ${ }^{5}$ y Antonio José Pérez Cortés ${ }^{2}$
}

\begin{abstract}
Resumen
El objetivo de esta investigación es adaptar y analizar las propiedades psicométricas del cuestionario Autoconcepto Forma 5 (AF-5) mediante técnicas de tipo exploratorio y confirmatorio en una población de estudiantes universitarios asiduos en el uso de videojuegos. La muestra está formada por 490 participantes de entre 20 y 29 años $(M=22.8$; DT=3.639), donde 297 son mujeres (60.6\%) y 193 hombres (39.4\%). El análisis factorial exploratorio se realizó mediante el programa FACTOR y el análisis factorial confirmatorio mediante el M-PLUS 7. Los resultados indican que el cuestionario ofrece índices de fiabilidad superiores a $\alpha=.70$. Se suprimió el factor familiar, al no alcanzar una fiabilidad en los constructos de la dimensión, por lo que se realizó el análisis confirmatorio con un modelo tetrafactorial. El coeficiente de correlación de Pearson revela que casi todos los factores presentan correlación significativa $(p<.01)$, siendo el valor más alto el establecido entre las dimensiones social y emocional.
\end{abstract}

Palabras clave: autoconcepto, universitarios, videojuegos, propiedades psicométricas

\begin{abstract}
The aim of this research is to adapt and analyze the psychometric properties of the AF-5 (Autoconcepto Forma 5) to a population of Spanish university students who play video games frequently. The employed techniques were an exploratory and confirmatory analysis. The sample consists of 490 participants with an age range between 20 and 29 years $(M=22.8, S D=3.639)$, being 297 subjects women $(60.6 \%)$ and 193 men (39.4\%). Exploratory factor analysis was performed using Factor Analysis program and confirmatory factor analysis using M-PLUS 7. Results show that the questionnaire provides higher reliability indices than $\alpha=.70$. The family factor was suppressed, as it did not reach reliability in the constructs of the dimension, so the confirmatory analysis was performed with a four-factor model.Thus, it was developed a confirmatory analysis with a factorial model of four options. Pearson correlation coefficient shows that almost all correlations between factors were significant $(p<.01)$ and that the association between social and emotional dimension was the strongest.
\end{abstract}

Keywords: self-concept, university students, video games, psychometric properties

\footnotetext{
Agradecimientos:

La presente investigación forma parte del Proyecto I+D PID 14-81, denominado "Programa de intervención educativa utilizando videojuegos activos y juegos motores para el apoyo a la docencia en materias de educación física y salud nutricional en los grados de primaria e infantil".

La presente investigación forma parte del Proyecto de Excelencia P11-TIC-7486, denominado "Videojuegos educativos para las aulas TIC: Metodología de desarrollo e implantación", financiado por la Consejería de Innovación, Ciencia y Empresa de la Junta de Andalucía.

La presente investigación forma parte del Proyecto DISPERSA TIN2015-67149-C3-R, denominado "Diseño de Juegos Pervasivos Basados en Experiencias de Aprendizaje Sensibles al Contexto", financiado por el Ministerio de Economía y Competitividad.

${ }^{1}$ Profesora del Departamento de Métodos de Investigación y Diagnóstico de la Educación de la Universidad de Granada, España. Correo: asuncionmm@ugr.es

2 (Correspondencia:) Profesor del Área de Corporal de la Universidad de Granada, España. Facultad de Ciencias de la Educación. Campus de Cartuja, España. Tel.: +34958248949. Correo: felixzo@ugr.es; antperez@ugr.es

${ }^{3}$ Profesor del Área de Corporal de la Universidad de Huelva, España.

${ }^{4}$ Profesor del Área de Didáctica de la Organización Escolar de la Universidad de Cádiz, España. Correo: tamara.espejo@uca.es

${ }^{5}$ Profesor del Área de Corporal de la Universidad de Almería, España. Correo: mcastros@ual.es
} 


\section{Introducción}

Resulta evidente la expansión tecnológica que se ha producido en todos los ámbitos de la sociedad, con especial énfasis en el académico (Wastiau, Blamire, Kearney, Quittre, Van de Gaer, \& Monseur, 2013). El uso de las Tecnologías de la Información y la Comunicación (TIC) en la esfera educativa avanza de forma exponencial, utilizándose como recurso didáctico y metodológico en todas las etapas (Prieto, Quiñones, Ramírez, Fuentes, Labrada, Pérez, \& Montero, 2011). De hecho, el uso de las TIC crece exponencialmente durante el periodo universitario con el fin de facilitar el proceso de aprendizaje, empleándose plataformas web para la gestión de archivos, redes sociales o incluso videojuegos (Figueiredo, 2012; Karpinski, Kirschner, Ozer, Mellott, \& Ochwo, 2013; Martínez-Martínez, 2013). Según Ballesteros, Cabero, Llorente, \& Morales (2010) este fenómeno se debe a que el estudiante universitario, al ser un nativo digital que ha nacido en el seno de la sociedad TIC (Karpinski et al., 2013), posee un abanico de competencias que le permite desenvolverse con total facilidad con este tipo de recursos, dotando de mayor operatividad al proceso de enseñanza (Bautista, Escofet, Forés, López, \& Marimon, 2013; Karpinski et al., 2013).

Numerosas investigaciones han focalizado sus esfuerzos en desarrollar el potencial educativo de los videojuegos (Revuelta \& Guerra, 2012). Estos dispositivos son juegos electrónicos que se visualizan a través de una pantalla y que pueden ser jugados a través de periféricos de diverso tipo, ya sea de forma activa o pasiva (Eguia, ContrerasEspinosa, \& Solano-Albajes, 2012; Chacón, Zurita, Castro-Sánchez, Espejo, MartínezMartínez, \& Linares, 2016). Sin embargo, el uso abusivo y patológico de los mismos puede relacionarse con consecuencias negativas a nivel físico, cognitivo y socio-afectivo, especialmente cuando su utilización alcanza niveles elevados desde la niñez y la adolescencia (Greenfield, 2014). En esta línea, estudios como el de Moncada, \& Chacón (2012) o Chacón, Zurita, Castro-Sánchez, Espejo, Martínez-Martínez, \& Pérez (2017), han demostrado la relación del uso de estos dispositivos con factores psicosociales, relacionándose con niveles bajos de conocimiento intrapersonal, autoestima, satisfacción con la vida o autoconcepto, constructo que ha sido estudiado en profundidad en la última década (Marsh, \& Martin, 2011; Zurita, Castro-Sánchez, Álvaro, Rodríguez, \& Pérez, 2016)

Según Hattie (2014), el autoconcepto se define como el conocimiento que un individuo posee de sí mismo a nivel físico, social, emocional, familiar y académico. Esta percepción difiere según la persona y es condicionada por la información externa que recibe y la experiencia vital del mismo (Fuentes, García, Gracia, \& Lila, 2011; Álvaro, 2015). De hecho, involucra una descripción objetiva y subjetiva, la cual integra diversos atributos que actúan como factores moduladores de los juicios que el sujeto fórmula (Salum-Fares, Marín, \& Vilariño, 2011).

El uso de los videojuegos se ha convertido en un fenómeno social en el siglo XXI (Eguia et al., 2012; Chacón, Castro-Sánchez, Muros-Molina, Espejo, Zurita, \& Linares, 2016), por lo que resulta de interés conocer la influencia que puede ejercer su utilización en el autoconcepto de cada individuo. Sobre la dimensión académica, varios estudios han demostrado que el ocio digital de pantalla se relaciona con el desplazamiento del tiempo de estudio, absentismo y una menor capacidad de atención en niños y adolescentes, mostrando algunos efectos negativos del uso de videojuegos (Marsh \& Martin, 2011; Franco, 2013; Inzunza et al., 2015). En este sentido, podría determinarse que el uso problemático de dispositivos de pantalla se asocia con peor rendimiento académico, y por tanto, con menores puntuaciones de la dimensión académica del autoconcepto (Puerta-Cortés \& Carbonell, 2013; Lloret, Cabrera, \& Sanz, 2015).

A nivel fisiológico, el uso de videojuegos implica altos niveles de sedentarismo que a largo plazo generan sobrepeso y obesidad (Villagrán, Rodríguez-Martín, Novalbos, Martínez, \& Lechuga, 2010), enfermedad que guarda una estrecha relación con problemas músculoesqueléticos, diabetes de tipo II o aumento del riesgo de padecer cáncer o muerte súbita (Beltrán, Devís, \& Peiró, 2012; Trost, Blair, \& Khan, 2014). En este sentido, el uso de dispositivos electrónicos de juego implicará puntuaciones más bajas en autoconcepto físico, ya que se asociará un peor estado de salud e imagen corporal (Marsh, 
Jowett, \& Lavalle, 2007; Zurita et al., 2016). De hecho, Chacón et al. (2016) demostraron como los escolares suelen tener menores niveles de autoestima y peor imagen corporal percibida cuando padecen problemas de peso ligados al ocio digital sedentario.

Álvaro (2015) establece que la dimensión social del autoconcepto se relaciona con la percepción que tiene la persona como ser social, implicando su autovaloración de la conducta en un contexto social concreto a través de sus habilidades sociales e interacciones con el prójimo. En una línea similar, la dimensión emocional de este constructo se relaciona con la capacidad que posee cada persona para percibir y comprender sus propias emociones y sentimientos (Fuentes et al., 2011; Hattie, 2014). Varios estudios han demostrado que el uso patológico de videojuegos se asocia con conductas antisociales como agresividad y competitividad, pobres habilidades sociales o mayores niveles de ansiedad, estrés, irritabilidad o insomnio, mostrando una posible vinculación con las dimensiones social y emocional del autoconcepto (Salum-Fares et al., 2011; Lancheros, Álvarez, Baquero, Amaya, \& Salazar, 2014; Espejo, Chacón, Castro-Sánchez, Martínez-Martínez, Zurita, \& Pinel, 2015).

No se halló mucha información relacionada con los videojuegos y el autoconcepto, aunque sí con elementos que influyen en sus dimensiones. En consecuencia, se encuentra en ésta área un campo fértil para la investigación, acerca de la cual discurre el presente documento. García, \& Musitu (1999) elaboraron un instrumento para determinar el autoconcepto en adolescentes, el cual se pretende adaptar a universitarios que juegan con videojuegos frecuentemente, dados sus efectos negativos en múltiples aspectos de la personalidad. El modelo de autoconcepto de García y Musitu (1999) divide este constructo en cinco dimensiones, sin embargo en la dimensión familiar disminuye en fortaleza en la etapa universitaria, por lo que se propone su supresión tal y como realizan en sus estudios Tomás, \& Oliver (2004), Núñez, Martín-Albo, Navarro, \& Grijalvo (2007) o Esnaola, Rodríguez y Goñi (2011), al no cargar las variables de esta dimensión de una manera adecuada, procediendo a la eliminación de factores en sus validaciones.
En definitiva, el objetivo del estudio ha sido analizar las propiedades psicométricas del cuestionario AF5 para calcular la fiabilidad del instrumento, adaptándolo y aplicándolo en una población universitaria que juega con videojuegos periódicamente. Para ello se ha realizado un análisis factorial exploratorio (AFE a partir de ahora) y un análisis factorial confirmatorio (AFC a partir de ahora).

\section{Método}

\section{Participantes}

La muestra de este trabajo de investigación está formada por 490 estudiantes universitarios de entre 20 y 29 años de edad $(M=22.8 ; D T=3.639)$, divididos en 297 mujeres (60.6\%) y 193 hombres (39.4\%). Según el hábito de consumo, el nivel de uso y atracción de los videojuegos, la muestra queda dividida en consumo bajo (81.2\%), consumo medio $(16.7 \%)$ y consumo alto $(2.0 \%)$. En la muestra se aplican los cuestionarios a 1.036 estudiantes universitarios de cuatro titulaciones de Humanidades de la Universidad de Granada. Se debe indicar que se detectaron 39 cuestionarios erróneos, que se eliminaron del estudio, así como 507 fueron excluidos por no jugar a los videojuegos.

\section{Instrumentos}

Los instrumentos administrados en esta investigación han sido dos, el cuestionario Autoconcepto Forma-5 (AF-5) de García \& Musitu (1999), este instrumento se fundamenta en el modelo teórico de Shavelson, Hubner y Stanton (1976), y está constituido por 30 preguntas agrupadas en cinco dimensiones: académico (ítems 1, 6, 11, 16, 21 y 26), social (ítems 2, 7, 12, 17, 22 y 27), emocional (ítems 3, 8, 13, 18, 23 y 28), familiar (ítems 4, 9, 14, 19, 24 y 29) y físico (ítems 5, 10, 15, 20, 25 y 30); es decir seis ítems por dimensión. Los participantes responden mediante una escala Likert de entre 1 y 5 puntos, donde el 1 es nunca y el 5 es siempre, esta forma de respuesta ha sido adaptada entre otros por García, Musitu, Riquelme, \& Riquelme (2011) y Salum-Fares et al. (2011), aunque en su origen la medición de los ítems oscilaba entre 1 y 99. En su versión original el test obtuvo una fiabilidad de $\alpha=.810$. 
También se utilizó el cuestionario CHCV de López (2012) para la valoración de hábitos de consumo de videojuegos. Este instrumento se estructura en 24 ítems; constituyendo 19 de ellos una única dimensión que representa el grado de atracción y uso de los videojuegos. Estos ítems son puntuados mediante una escala de tipo Likert de cinco opciones ( $1=$ Nada de acuerdo; $5=$ Totalmente de acuerdo), realizándose una sumatoria final que indica una menor o mayor dependencia hacia estos dispositivos. Finalmente, la muestra fue clasificada en terciles en función de la puntuación obtenida según el nivel de uso y atracción de los videojuegos (bajo, medio y alto).

\section{Procedimiento}

Los cuestionarios se administraron en horario lectivo y se aplicaron de manera grupal por los investigadores, preparados para tal efecto. Los participantes desconocen la finalidad del estudio con el fin de evitar respuestas no sinceras y reducir lo máximo posible el efecto de deseabilidad social.

\section{Análisis de los Datos}

Este estudio analiza las propiedades psicométricas (fiabilidad, análisis factorial exploratorio y análisis factorial confirmatorio) del instrumento AF5 mediante los paquetes estadísticos SPSS 22.0, FACTOR Analysis 9.3.1 (Lorenzo-Seva \& Ferrando, 2006) y M-Plus 7 (Muthén \& Muthén, 2007). En primer lugar, se utiliza SPSS 22.0 para analizar las propiedades métricas de cada ítem, empleando coeficientes descriptivos básicos (media, dispersión, curtosis y asimetría). En segundo lugar, se emplea FACTOR para determinar la bondad de ajuste y validez de la escala (Bentler, 1990; McDonal \& Marsh, 1990). Finalmente, se utilizó alpha de Cronbach y MPLUS 7 para verificar la consistencia interna del instrumento (Muthén \& Muthén, 2007).

\section{Resultados}

En esta primera parte del análisis se han calculado los valores descriptivos del estudio, y siguiendo los pasos recomendados por expertos (Schmider, Ziegler, Danay, Beyer, \& Bühner, 2010), se ha tomado la decisión de prescindir totalmente de los ítems que componen la dimensión familiar del autoconcepto (V.4, V.9, V.14, V.19, V.24 y V.29) en el instrumento AF5 aplicado en universitarios. Esto es debido a que las pruebas de dispersión (asimetría y curtosis) presentan valores superiores a 2.00 .

\section{Descriptivos de los ítems de del AF5}

Previo a la realización del AFE se ha tomado la decisión de eliminar las variables que componen la dimensión familiar del instrumento, pues los valores de la curtosis y asimetría fueron más elevados de lo normal. Seguidamente, mediante la utilización del programa FACTOR Analysis (Lorenzo-Seva, \& Ferrando, 2006) se han rotando cuatro factores. El estadístico de Bartlett, [4242.9 (df=276; $p=.000010)]$ y el test de Kaiser-Meyer-Olkin $(\mathrm{KMO})=.81$, utilizados para probar si la muestra procede de poblaciones con la misma varianza y si presenta una buena adecuación muestral, indican un buen ajuste de los datos para ser sometidos al análisis factorial. Los cuatro factores extraídos explican el $52 \%$ de la varianza total, el índice de bondad de ajuste (GFI) fue de .98 y la raíz cuadrática media de los residuales (RMSR) de .040. Todos estos datos nos indican un buen ajuste para estos ítems.

Como se determina en la Tabla 2, ha desaparecido una variable "V.15" por presentar valores menores de .30. La escala definitiva ha quedado formada por cuatro factores. El primero, conformado por seis variables, se corresponde con la dimensión social; el segundo factor, constituido por seis variables, se relaciona con la dimensión académica; un tercer factor, donde se agrupan cinco variables, coincide con la dimensión física; y por último el cuarto factor, constituido por seis variables, se corresponde con la dimensión emocional.

A continuación se muestra la configuración del cuestionario con un buen ajuste. El coeficiente de fiabilidad del cuestionario fue de .823 , mientras que por factores se obtuvo un .769 para el factor 1 (social), .773 para el factor 2 (académico), .778 para el factor 3 (físico) y .815 en el factor 4 (emocional).

Como se observa en la Tabla, se obtuvo un alpha de Cronbach satisfactorio en su totalidad y en cada uno de los factores, presentando valores $p>$.70. Al aplicar el coeficiente de correlación de Pearson, se aprecia que casi todos los factores 
Tabla 1. Descriptivos de los ítems de del AF5

\begin{tabular}{lcccccc}
\hline & Media & DT & Varianza & Asimetría & Curtosis & Rango \\
\hline V 01 & 4.20 & .582 & .339 & -.183 & .269 & 3 \\
V 02 & 4.11 & .842 & .709 & -.772 & .272 & 4 \\
V 03 & 2.76 & 1.008 & 1.015 & .199 & -.478 & 4 \\
V 04 & 4.25 & .998 & .995 & -1.393 & 1.302 & 4 \\
V05 & 3.74 & .950 & .902 & -.474 & -.155 & 4 \\
V06 & 3.62 & .779 & .607 & -.302 & .556 & 4 \\
V 07 & 4.45 & .615 & .379 & -.802 & .451 & 3 \\
V 08 & 2.74 & 1.058 & 1.119 & .014 & -.718 & 4 \\
V 09 & 4.47 & .738 & .544 & -1.374 & 1.502 & 3 \\
V 10 & 3.46 & 1.237 & 1.529 & -.394 & -.851 & 4 \\
V 11 & 3.51 & .932 & .868 & -.434 & .214 & 4 \\
V 12 & 4.23 & 1.036 & 1.073 & -1.337 & .981 & 4 \\
V 13 & 3.73 & 1.018 & 1.037 & -.592 & -.156 & 4 \\
V 14 & 4.77 & .562 & .316 & -2.781 & 8.581 & 4 \\
V 15 & 3.65 & .956 & .915 & -.507 & .197 & 4 \\
V 16 & 3.44 & .847 & .717 & -.298 & .458 & 4 \\
V 17 & 4.48 & .662 & .438 & -1.114 & .940 & 3 \\
V 18 & 3.59 & 1.058 & 1.118 & -.487 & -.402 & 4 \\
V 19 & 4.76 & .602 & .362 & -3.011 & 10.335 & 4 \\
V 20 & 3.72 & 1.040 & 1.082 & -.708 & .072 & 4 \\
V21 & 3.90 & .753 & .567 & -.471 & .455 & 4 \\
V 22 & 3.68 & 1.131 & 1.280 & -.504 & -.683 & 4 \\
V 23 & 2.92 & 1.199 & 1.438 & -.007 & -.921 & 4 \\
V 24 & 4.48 & .839 & .704 & -1.802 & 3.256 & 4 \\
V 25 & 3.78 & 1.085 & 1.176 & -.587 & -.397 & 4 \\
V 26 & 3.63 & .809 & .654 & -.237 & .195 & 4 \\
V 27 & 4.08 & .905 & .818 & -.882 & .707 & 4 \\
V 28 & 3.50 & 1.063 & 1.130 & -.462 & -.366 & 4 \\
V 29 & 4.76 & .639 & .408 & -3.236 & 11.609 & 4 \\
V 30 & 3.52 & .968 & .937 & -.219 & -.268 & 4 \\
\hline & & & & & & 4 \\
\hline
\end{tabular}

Tabla 2. Matriz Factorial Rotada

\begin{tabular}{|c|c|c|c|c|c|c|c|c|}
\hline \multirow[t]{2}{*}{ Variables } & \multicolumn{4}{|c|}{ Matriz Factorial Rotada } & \multicolumn{4}{|c|}{$\begin{array}{c}\text { Matriz Factorial Rotada } \\
\text { (omitidas las cargas inferiores a } \\
.300 \text { ) } \\
\end{array}$} \\
\hline & F1 & F2 & F3 & F4 & F1 & $\mathrm{F} 2$ & F3 & $\mathrm{F} 4$ \\
\hline V 01 & .022 & .442 & .090 & -.100 & & .442 & & \\
\hline V 02 & .891 & -.086 & -.027 & -.059 & .891 & & & \\
\hline V 03 & -.042 & -.097 & .173 & .539 & & & & .539 \\
\hline V 05 & .002 & .068 & .620 & -.069 & & & .620 & \\
\hline V 06 & -.036 & .757 & -.001 & -.011 & & .757 & & \\
\hline V 07 & .670 & .087 & .061 & -.156 & .670 & & & \\
\hline V 08 & -.109 & .003 & -.017 & .754 & & & & .754 \\
\hline V 10 & .048 & -.162 & .738 & -.058 & & & .738 & \\
\hline V 11 & .078 & .303 & .042 & -.167 & & .303 & & \\
\hline V 12 & .739 & -.165 & -.101 & .144 & .739 & & & \\
\hline V 13 & .046 & -.108 & .067 & .533 & & & & .533 \\
\hline V 15 & .141 & .297 & .285 & -.024 & & & & \\
\hline V 16 & .080 & .587 & -.111 & .089 & & .587 & & \\
\hline V 17 & .361 & .133 & .064 & -.065 & .361 & & & \\
\hline V 18 & -.012 & -.000 & .004 & .709 & & & & .709 \\
\hline V 20 & -.003 & .143 & .501 & .202 & & & .501 & \\
\hline V 21 & -.042 & .720 & .035 & .001 & & .720 & & \\
\hline V 22 & .509 & -.046 & -.155 & .225 & .509 & & & \\
\hline V 23 & .109 & .044 & -.022 & .629 & & & & .629 \\
\hline V 25 & -.097 & -.118 & .873 & -.082 & & & .873 & \\
\hline V 26 & -.090 & .829 & .007 & .013 & & .829 & & \\
\hline V 27 & .537 & .038 & .235 & -.074 & .537 & & & \\
\hline V 28 & .028 & .100 & -.014 & .693 & & & & .693 \\
\hline V 30 & .049 & .195 & .458 & .136 & & & .458 & \\
\hline
\end{tabular}


presentan correlación significativa en un 99\%, $(p<.01)$, siendo el valor más alto el establecido al relacionarse la dimensión emocional con la dimensión social (.336). También presentan correlaciones significativas el factor social con el físico y académico; asimismo el factor físico también correlaciona significativamente con la dimensión social y emocional, y la académica con la emocional.

Tabla 3. Carga factorial de las dimensiones del

\begin{tabular}{|c|c|c|c|c|}
\hline \multicolumn{5}{|c|}{ AF5 } \\
\hline Variables & F1 & $\mathrm{F} 2$ & F3 & F4 \\
\hline $\mathrm{V} \quad 02$ & .891 & & & \\
\hline V 07 & .670 & & & \\
\hline V 12 & .739 & & & \\
\hline V 17 & .361 & & & \\
\hline V 22 & .509 & & & \\
\hline V 27 & .537 & & & \\
\hline V 01 & & .442 & & \\
\hline V 06 & & .757 & & \\
\hline V 11 & & .303 & & \\
\hline V 16 & & .587 & & \\
\hline V 21 & & .720 & & \\
\hline V 26 & & .829 & & \\
\hline V 05 & & & .620 & \\
\hline V 10 & & & .738 & \\
\hline V 20 & & & .501 & \\
\hline V 25 & & & .873 & \\
\hline V 30 & & & .458 & \\
\hline V 03 & & & & .539 \\
\hline V 08 & & & & .754 \\
\hline V 13 & & & & .533 \\
\hline V 18 & & & & .709 \\
\hline V 23 & & & & .629 \\
\hline V 28 & & & & .673 \\
\hline Alfa (.823) & .769 & .773 & .778 & .815 \\
\hline
\end{tabular}

Tabla 4. Correlación delas dimensiones del AF5

\begin{tabular}{lcccc}
\hline & Factor 1 & Factor 2 & Factor 3 & Factor 4 \\
\hline $\begin{array}{l}\text { Factor1: Dimensión } \\
\text { Social }\end{array}$ & 1 & & & \\
$\begin{array}{l}\text { Factor2: Dimensión } \\
\text { Académico }\end{array}$ & $.210^{* *}$ & 1 & & \\
$\begin{array}{l}\text { Factor3: Dimensión } \\
\text { Físico }\end{array}$ & $.304^{* *}$ & $.137^{* *}$ & 1 & \\
$\begin{array}{l}\text { Factor4: Dimensión } \\
\text { Emocional }\end{array}$ & $.336^{* *}$ & .014 & $.273^{* *}$ & 1 \\
\hline
\end{tabular}

**. La correlación es significativa al nivel .01 (bilateral).

*. La correlación es significante al nivel .05 (bilateral).

Para finalizar, empleando el M-PLUS, se ha realizado un AFC con resultados favorables y aceptables, tanto para el RMSEA (Root Mean Square Error of Approximation) como para el CFI (Comparative Fit Index) y el TLI (Tucker-Lewis Index), siendo los valores alcanzados en el RMSEA de .065 y los CFI y TLI de .90 y .84 , si bien este último no alcanza el valor recomendado de .90 , este índice penaliza los modelos que incluyen parámetros con poca contribución al ajuste (Brown, 2006), pero puesto que todos los parámetros estimados resultan ser estadísticamente significativos, se juzga correcto mantenerlos en el modelo.

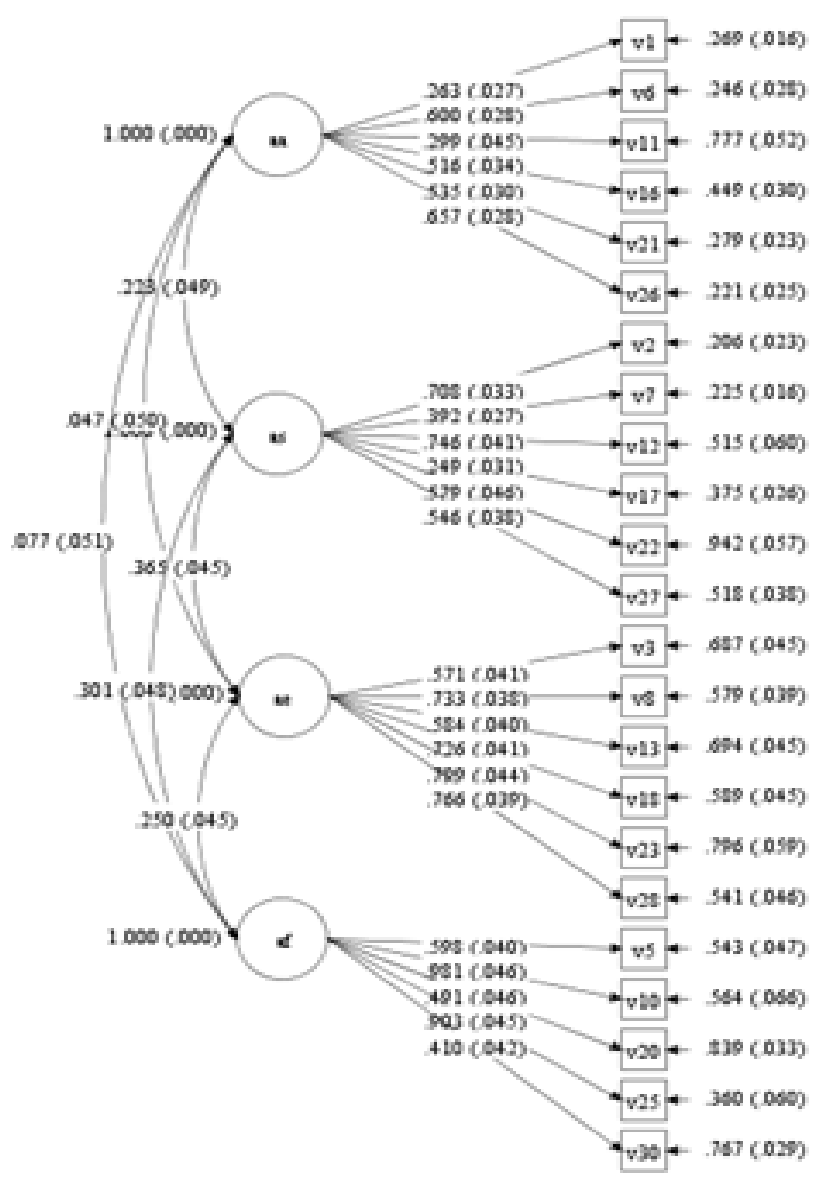

Figura 1. Correlación Distribución de los factores del AF-5

\section{Discusión}

A la hora de realizar cualquier evaluación psicológica se considera imprescindible disponer de cuestionarios que reporten datos fiables de lo que se quiere medir, por lo que el principal objetivo de este trabajo ha sido analizar las propiedades psicométricas del cuestionario AF-5 y observar su adaptación y aplicación en estudiantes universitarios que juegan frecuentemente con videojuegos.

El colectivo objeto de estudio reúne unas características que lo diferencian del resto, se consideran nativos digitales por nacer en el seno de la sociedad TIC y poseer unas competencias y capacidades mejores a las de sus homólogos de mayor edad en el uso de nuevas tecnologías 
(Ballesteros et al., 2010; Karpinski et al., 2013). Este sector hace un uso elevado de dispositivos de pantalla, y más específicamente de videojuegos, cuyo uso patológico puede relacionarse con problemas de tipo físico, cognitivo y socioafectivo (Greenfield, 2014).

Los resultados obtenidos han sido satisfactorios en cuanto a los coeficientes alpha de Cronbach, tanto en la totalidad de la escala como para los cuatro factores, mostrando que es un instrumento válido y fiable para establecer el autoconcepto de un modo multidimensional, incluso prescindiendo del factor familiar. Para entender esta idea se debe partir de las apreciaciones citadas por González-Pienda et al., (2000) y Goñi y Fernández (2007) en cuanto a los efectos en el campo social, académico y emocional en relación al contexto familiar. En el marco de este trabajo queda eliminado el factor familiar, mientras en otros trabajos sí que aparece al quedar demostrada la importancia de esta dimensión, y especialmente en la etapa adolescente (Molero, Ortega-Álvarez, Valiente, \& Zagalaz-Sánchez, 2010).

Los resultados se mantienen estables en todas las dimensiones, cargando los factores de una forma coherente. Asimismo, y aunque este test ha sido ampliamente aplicado en el contexto universitario (Martín-Monzón, 2007; Gargallo, Garfella, Sánchez, Ros, \& Serra, 2009; Álvarez, Cuevas, Lara, \& González, 2015; Rojas \& Bolivar, 2015), no lo había sido nunca en estudiantes universitarios asiduos en el uso de videojuegos. Como ya se ha citado, los datos obtenidos apoyan la teoría de la multidimensionalidad con la omisión del factor familiar, presentando unos índices de ajuste aceptable y con una buena consistencia interna y estabilidad temporal, revelando valores que superan el .70 y que replica el modelo utilizado a partir de la base teórica propuesta por Shavelson et al. (1976), ajustándose de manera correcta en cuatro dimensiones y obteniendo una adecuada fiabilidad similar a otros estudios previos (Tomás \& Oliver, 2004; Murgui, García, García, \& García, 2012). Asimismo, las cifras arrojadas sugieren algunas cuestiones que deberían ser atendidas en futuros trabajos.

En este estudio se encontró correlación entre la dimensión social y el resto de dimensiones, siendo la emocional la que mostraba asociaciones de mayor fortaleza, si bien todas ellas eran de menor magnitud, se entiende que en esta etapa, las personas jóvenes están caracterizadas por un aumento de las relaciones sociales, por lo que esta correlación con la dimensión emocional, física y académica puede estar condicionada por el uso abusivo de los videojuegos, que hacen a las personas más inactivas físicamente, pudiéndose denominar como sedentarios tecnológicos (Castro-Sánchez, Martínez-Martínez, Zurita, Chacón, Espejo, \& Cabrera, 2015), introvertidos (Espejo et al., 2015) y con peores calificaciones académicas (Dezcallar, Clariana, Cladellas, Badia, \& Gotzens, 2014). La direccionalidad negativa que a veces adquiere el ocio digital se puede relacionar con una vivencia no positiva de las relaciones sociales, emocionales, físicas y académicas, no exclusivamente desde el punto de vista de la persona sino también de la sociedad (Salum-Fares et al., 2011; Espejo et al., 2015; Chacón et al., 2016).

Tal y como establecen García y Musitu (1999), todos los factores del instrumento AF-5 reportan datos concisos sobre la percepción que un sujeto posee de sí mismo. Sin embargo, este estudio explica como la dimensión familiar puede ser excluida cuando este instrumento se aplica en estudiantes universitarios. Justificando estas premisas, Martínez-Martínez (2013) recuerda como la dimensión familiar no se encuentra muy definida en el colectivo universitario, pues este sector se encuentra en un periodo de emancipación familiar e incluso en fase de independencia financiera que otorga a la estructura familiar un segundo plano (Arce, Fariña, Novo, \& Seijo, 2012; Richardson, Abraham, \& Bond, 2012)

Desde el punto de vista metodológico, el modelo obtenido apoya el uso de la medida global del autoconcepto hacia el colectivo de universitarios, si bien presenta algunas limitaciones. En primer lugar, la muestra del estudio ha sido no probabilística y por tanto los resultados no pueden ser generalizados a toda la población española jugadora de videojuegos. Además, en futuros trabajos debería explorarse la estructura de esta población en función del tipo de videojuegos que utilizan. En segundo lugar, se podría utilizar para establecer las posibles 
diferencias con otras variables (p. ej., género, logros académicos o tipo de titulación). No obstante, podría decirse que los resultados de este estudio apoyan la idea del AF-5 como test válido y fiable para medir de forma satisfactoria el autoconcepto en estudiantes universitarios que emplean videojuegos de forma asidua, permitiendo el análisis de este constructo de forma multidimensional (excluyendo el componente familiar).

\section{Referencias}

Arce, R., Fariña, F., Novo, M., \& Seijo, D. (2012). Efecto del sistema de enseñanza en el rendimiento académico, burnout experimentado y estrés académico. Aula Abierta, 40(2), 3-10.

Álvarez, L., Cuevas, R., Lara, A., \& González, J. (2015). Diferencias del autoconcepto físico en practicantes y no practicantes de actividad física en estudiantes universitarios. Cuadernos de Psicología del Deporte, 15(2), 27-34.

Álvaro, J. I. (2015). Análisis del autoconcepto en relación con factores educativos, familiares, físico y psicosociales en adolescentes de la provincia de Granada. Tesis Doctoral: Universidad de Granada.

Ballesteros, C., Cabero, J., Llorente, M. C., \& Morales, J. A. (2010). Usos del e-learning en las universidades andaluzas: Estado de la situación y análisis de buenas prácticas. PixelBit, 37, 7-18.

Bautista, G., Escofet, A., Forés, A., López, M., \& Marimon, M. (2013). Superando el concepto de nativo digital. Análisis de las prácticas digitales del estudiantado universitario. Digital Education Review, 24, 1-22.

Beltrán, V. J., Devís, J., \& Peiró, C. (2012). Actividad física y sedentarismo en adolescentes de la comunidad valenciana. Revista Internacional de Medicina y Ciencias de la Actividad Física y del Deporte, 12(45), 123-137.

Bentler, P. M. (1990). Comparative fit indexes in structural models. Psychological Bulletin, 107, 238-246.

Brown, T. (2006). Confirmatory factor analysis for applied research. New York: The Guilford Press.
Castro-Sánchez, M., Martínez-Martínez, A., Zurita, F., Chacón, R., Espejo, T., \& Cabrera, A. (2015). Uso de videojuegos y su relación con las conductas sedentarias en una población escolar y universitaria. Journal for Educators, Teachers and Trainers, 6(1), 40-51.

Castro-Sánchez, M., Chacón, R., \& Padial, R. (2017). Exergames y discapacidad. ESHPAEducation, Sport, Health and Physical Activity, 1(1), 2-16.

Chacón, R., Castro-Sánchez, M., Muros-Molina, J. J., Espejo, T., Zurita, F., \& Linares, M. (2016). Adhesión a la dieta mediterránea en estudiantes universitarios y su relación con los hábitos de ocio digital. Nutrición Hospitalaria, 33, 405-410. doi:10.20960/nh.124

Chacón, R., Zurita, F., Castro-Sánchez, M., Espejo, T., Martínez-Martínez, A., \& Linares, M. (2016). Estudio sobre la aplicabilidad de exergames para la mejora de los índices de obesidad y la imagen corporal en escolares. Revista Iberoamericana de Psicología del Ejercicio y el Deporte, 11(1), 97-105.

Chacón, R., Zurita, F., Castro-Sánchez, M., Espejo, T., Martínez-Martínez, A., \& Pérez, A. J. (2017). Clima motivacional hacia el deporte y su relación con hábitos de ocio digital sedentario en estudiantes universitarios. Saude e Sociedades, 26(1), 2939. doi:10.1590/S0104-12902017166561

Dezcallar, T., Clariana, M., Cladellas, R., Badia, M., \& Gotzens, C. (2014). La lectura por placer: Su incidencia en el rendimiento académico, las horas de televisión y las horas de videojuegos. Ocnos: Revista de Estudios sobre Lectura, 12, 107-116. doi:10.18239/ocnos_2014.12.05

Eguia, J. L., Contreras-Espinosa, R. S., \& SolanoAlbajes, L. (2012). Videojuegos: Conceptos, historia y su potencial como herramientas para la educación. 3C TIC, 2, 1-14.

Esnaola, I., Rodríguez, A., \& Goñi, E. (2011). Propiedades psicométricas del cuestionario de Autoconcepto AF5. Anales de Psicología, 27(1), 109-117.

Espejo, T., Chacón, R., Castro-Sánchez, M., Martínez-Martínez, A., Zurita, F., \& Pinel, C. (2015) Análisis descriptivo del uso problemático y hábitos de consumo de los videojuegos con relación al género en 
estudiantes universitarios. RELATEC, 14(3), 85-93. doi:10.17398/1695-288X.14.3.85

Figueiredo, A. (2012). Características psicométricas da adaptação portuguesa do perfil de auto-percepção para estudantes universitários - $\quad$ SPPCS. Revista Iberoamericana de Diagnóstico y Evaluación - e Avaliação Psicológica, 33, 93-110.

Franco, A. A. (2013). El uso de la tecnología: Determinación del tiempo que los jóvenes de entre 12 y 18 años dedican a los equipos tecnológicos. RIED, 16(2), 107-125.

Fuentes, M. C., García, J. F., Gracia, E., \& Lila, M. (2011). Autoconcepto y ajuste psicosocial en la adolescencia. Psicothema, 23(1), 7-12.

García, F., \& Musitu, G. (1999). AF5: Autoconcepto Forma 5. Madrid: TEA Ediciones.

García, F., Musitu, G., Riquelme, E., \& Riquelme, P. (2011). A confirmatory factor analysis of the "Autoconcepto Forma 5" questionnaire in young adults from Spain and Chile. Spanish Journal of Psychology, 14, 648-658. doi:10.5209/rev_SJOP.2011.v14.n2.13

Gargallo, B., Garfella, P., Sánchez, F., Ros, C., \& Serra, B. (2009). La influencia del autoconcepto en el rendimiento académico en estudiantes universitarios. Revista REOP, 20(1), 16-28.

González-Pienda, J.A., Núñez, J.C., GonzálezPumariega, S., Álvarez, L., Roces, C., García, M., González, P., Cabanach, R. G., \& Valle, A. (2000). Autoconcepto, proceso de atribución causal y metas académicas en niños con o sin dificultades de aprendizaje. Psicothema, 12(4), 548-556.

Goñi, E., \& Fernández, A. (2007). Los dominios social y personal del autoconcepto. Revista de Psicodidáctica, 12(2), 179-194.

Greenfield, P. M. (2014). Mind and media: The effects of television, video games, and computers. Psychology Press: New York.

Hattie, J. (2014). Self-concept. Psychology Press: New York.

Inzunza, B., Ortíz, L., Pérez, C., Torres, G., McColl, P., Meyer, A., ..., \& Bustamante, C. (2015). Estructura Factorial y Confiabilidad del Cuestionario de Satisfacción Académica en Estudiantes de Medicina Chilenos. Revista
Iberoamericana de Diagnóstico y Evaluación - e Avaliação Psicológia, 40, 73-82.

Karpinski, A. C., Kirschner, P. A., Ozer, I., Mellott, J. A., \& Ochwo, P. (2013). An exploration of social networking site use, multitasking and academic performance among United States and European University students. Computers in Human Behavior, 29(3), 1182-1192. doi:10.1016/j.chb.2012.10.011

Lancheros, M. L., Álvarez, C. F., Baquero, L. A., Amaya, M. A., \& Salazar, C. A. (2014). Incidencia de videojuegos en el retraimiento de niños de 6 a 12 años. Revista de Educación y Humanismo, 16(27), 15-26.

Lloret, D., Cabrera, V., \& Sanz, Y. (2015). Relaciones entre hábitos de uso de videojuegos, control parental y rendimiento escolar. EJIHPE, 3(3), 237-248. doi:10.1989/ejihpe.v3i3.46

López, F. (2012). Construcción y validación de un cuestionario sobre los hábitos de consumo de videojuegos en preadolescentes. EDUTEC, $40,1-12$.

Lorenzo-Seva, U., \& Ferrando, P. J. (2006). FACTOR: A computer program to fit the exploratory factor analysis model. Behavioral Research Methods, Instruments and Computers, 38(1), 88-91. doi:10.3758/BF03192753

Marsh, H., \& Martin, A. (2011). Academic selfconcept and academic achievement: Relations and causal ordering. British Journal of Educational Psychology, 81(1), 59-77. doi:10.1348/000709910X503501

Marsh, H., Jowett, S., \& Lavalle, D. (2007). Physical self-concept and sport. Social Psychology in Sport, 159-179.

Martínez-Martínez, A. (2013). La Orientación como actividad educativa y vocacional en los itinerarios curriculares del alumnado de Bachiller y Formación Profesional y su inclusión en el mercado laboral. Tesis Doctoral: Universidad de Granada.

Martín-Monzón, I. M. (2007). Estrés académico en estudiantes universitarios. Apuntes de Psicología, 25(1), 87-99.

McDonald, R. P., \& Marsh, H. W. (1990). Choosing a multivariate model: Noncentrality and goodness of fit. Psychological Bulletin, 107, 247-255. 
Molero, D., Ortega-Álvarez, F., Valiente, I., \& Zagalaz-Sánchez, M. L. (2010). Estudio comparativo del autoconcepto físico en adolescentes en función del género y del nivel de actividad físico-deportiva. Retos. Nuevas tendencias en Educación Física, Deporte y Recreación, 17,38-41. doi:10.1037/0033-2909.107.2.247

Moncada, J., \& Chacón, Y. (2012). El efecto de los videojuegos en variables sociales, psicológicas y fisiológicas en niños y adolescentes. Retos Nuevas tendencias en Educación Física, Deporte y Recreación, 21, 43-49.

Murgui, S., García, C., García, A., \& García, F. (2012). Autoconcepto en jóvenes practicantes de danza y no practicantes: Análisis factorial confirmatorio de la escala AF5. Revista de Psicología del Deporte, 21(2), 263-269.

Muthén, L. K., \& Muthén, B. O. (2007). Mplus. Statistical analysis with latent variables. Version, 3.

Núñez, J. L., Martín-Albo, J., Navarro, J. G., \& Grijalvo, F. (2007). Análisis de las propiedades psicométricas del cuestionario Autoconcepto Forma 5 en estudiantes universitarios. Estudios de Psicología, 28(3), 333-342. doi:10.1174/021093907782506461

Prieto, V., Quiñones, I., Ramírez, G., Fuentes, Z., Labrada, T., Pérez, O., \& Montero, M. (2011). Impacto de las tecnologías de la información y la comunicaciones en la educación y nuevos paradigmas del enfoque educativo. Educación Médica Superior, 25(1), 95-102.

Puerta-Cortés, D. X., \& Carbonell, X. (2013). Uso problemático de Internet en una muestra de estudiantes universitarios colombianos. Avances en Psicología Latinoamericana, 31(3), 620-631.

Revuelta, F. I., \& Guerra, J. (2012). ¿Qué aprendo con videojuegos? Una perspectiva de metaaprendizaje del videojugador. Revista de Educación a Distancia, 33(15), 1-25.

Richardson, M., Abraham, C., \& Bond, R. (2012). Psychological correlates of university students academic performance: A systematic review and meta-analysis. Psychological Bulletin, 138(2), 353-387. doi:10.1037/a0026838

Rojas, F. F., \& Bolivar, J. M. (2015). Autoconcepto estudiantil y modalidades de enseñanza a distancia (B-Learning y $\mathrm{E}$ learning). Paradígma, 30(2), 99-112.

Salum-Fares, A., Marín, A. R., \& Reyes, A. C. (2011). Relevancia de las dimensiones del Autoconcepto en estudiantes de Escuelas secundarias de Ciudad Victoria, Tamalulipas. Revista Electrónica de Psicología Iztacala, 14(2), 255-272.

Schmider, E., Ziegler, M., Danay, E., Beyer, L., \& Bühner, M. (2010). Is it really robust? Reinvestigating the robustness of ANOVA against violations of the normal distribution assumption. Methodology, 6, 147-151. doi:10.1027/1614-2241/a000016

Shavelson, J., Hubner, J. J., \& Stanton, G. C. (1976). Self-concept: validation of construct interpretations. Review of Educational Research, 46, 407-442. doi:10.3102/00346543046003407

Tomás, J. M., \& Oliver, A. (2004). Confirmatory factor analysis of a Spanish multidimensional scale of self-concept. Revista Interamericana de Psicología, 38, 285-293.

Trost, S., Blair, S., \& Khan, K. (2014). Physical inactivity remains the greatest public health problems of the 21st century: Evidence, improved methods and solutions using the " 7 investments that works as a framework. British Journal of Sports Medicine, 48(3), 169-170. doi:10.1136/bjsports-2013-093372

Villagrán, S., Rodríguez-Martín, A., Novalbos, J. P., Martínez, J. M., \& Lechuga, J. L. (2010). Hábitos y estilos de vida modificables en niños con sobrepeso y obesidad. Nutrición Hospitalaria, 25(5), 823-831. doi:10.3305/nh.2010.25.5.4683

Wastiau, P., Blamire, R., Kearney, C., Quitre, V., Van de Gaer, E., \& Monseur, C. (2013). The use of ICT in education: A survey of schools in Europe. European Journal of Education, 48(1), 11-27. doi: 10.1111/ejed.12020

Zurita, F., Castro-Sánchez, M., Álvaro, J. I. Rodríguez, S., \& Pérez, A. J. (2016). Autoconcepto, actividad física y familia: Análisis de un modelo de ecuaciones estructurales. Revista de Psicología del Deporte, 25(1), 97-104. 